\title{
A signal decomposition method based on repeated extraction of maximum energy component for offshore structures
}

\author{
Fushun Liu, ${ }^{\mathrm{a}, *}$, Shujian $\mathrm{Gao}^{\mathrm{a}}$, Dianzi Liu ${ }^{\mathrm{b}}, \mathrm{Hu}_{\mathrm{Zhou}}^{\mathrm{c}}$ \\ ${ }^{a}$ Shandong Province Key Laboratory of Ocean Engineering, Ocean University of China, Qingdao 266100, China \\ ${ }^{b}$ Engineering, Faculty of Science, University of East Anglia, Norwich, NR4 7TJ, UK \\ ${ }^{c}$ Huadong Engineering Corporation Limited, PowerChina, Hangzhou 311122, China
}

\begin{abstract}
Contrary to most signal decomposition methods that usually decompose an original signal into a series of components simultaneously, a novel approach based on repeated extraction of Maximum Energy Component (MEC) is proposed. The approach starts from determination of the MEC referring to the estimated Power Spectral Density (PSD) function, and then represents the MEC by employing an exponential function to fit the original signal. By defining a stopping criterion based on two adjacent estimated PSDs, each MEC can be accurately extracted with an improved performance throughout the entire signal decomposition. To verify the proposed method, a single degree-of-freedom system subject to harmonic loads has been examined. Numerical results show that the analytical response can not only be decomposed into four MECs corresponding to the excitation and the system, respectively, but also provide an accurate estimation of natural frequency and damping ratio of the system. Meanwhile, by observing results from the Ensemble Empirical Mode Decomposition (EEMD), Variational Mode Decomposition (VMD) and Prony based on state-space model (Prony-SS), an improved decomposition accuracy has been achieved from the proposed approach. Furthermore, experimental data from the Norwegian Deepwater Programme and two sets of field-test data from one fixed offshore platform and an offshore wind turbine have been used to demonstrate the correctness of the developed signal decomposition method. It is noted that divergence in results by Prony-SS can be observed when a very large model order is used, while the proposed method provides the better decomposition and reconstruction of signals.
\end{abstract}

Keywords: offshore structures; dynamic analysis; signal decomposition; maximum energy component.

\section{Nomenclature}

\footnotetext{
${ }^{*}$ Corresponding author: percyliu@ ouc.edu.cn (Fushun Liu).
} 
A

$b_{p}$

$c_{i, n}$

$\bar{c}_{i}$

$f_{n}^{t}(t), f_{n}^{s}(t)$

$f_{n}(t)$

$f_{\text {rem }}$

F

H

$N_{n o i}, N_{p}, N_{f}$

$N_{r}, N_{c}$

$N_{t}, N_{s}$

$R_{f}(t)$

Re, Im

$s$

$S C_{s w}$

$S_{f}(\tilde{\omega})$

$\mathbf{S}_{\text {od }}^{f}$

$\Delta t$

$T$

$\mathbf{U}_{o d}^{f}, \mathbf{V}_{o d}^{f}$

$y, y_{n}, \bar{y}$

$z_{n}$

$\alpha_{n}$

$\lambda_{n}$

$\omega_{d}$

$\rho_{n}, \xi_{n}, \omega_{n}, \theta_{n}$

$\bar{\rho}, \bar{\xi}, \bar{\omega}, \bar{\theta}$

$\tilde{\rho}_{n}, \tilde{\xi}_{n}, \tilde{\omega}_{n}, \tilde{\theta}_{n}$ state matrix defined in state-space model.

$p$ th coefficient in the Prony-SS method.

$n$th IMF under $i$ th EMD.

$i$ th IMF of the EEMD.

transient and steady-state responses.

total responses of a structure.

remaining part of the original signal.

similarity transformation of a coefficient matrix of the converted ODEs.

Hankel matrix.

number of adding white noise, sampled points and components.

selected number of rows and columns in $\mathbf{H}$.

number of components in transient and steady-state responses.

autocorrelation function of $f_{n}(t)$.

real and imaginary parts of a complex number.

Laplace variable.

stopping criterion.

spectral density function.

singular matrix with a dimension of $o d \times o d$.

time interval.

transpose operator of a vector.

singular vectors with a dimension of $N_{r} \times o d$.

original signal, added Gaussian white noise and overall signal in EMD.

roots of a characteristic equation.

coefficient of white noise.

$\lambda_{n}=\frac{\ln z_{n}}{\Delta t}$.

damped frequency.

amplitude, damping ratio, natural frequency and phase, embedded in transient response.

amplitude, damping ratio, natural frequency and phase, embedded in steady-state response.

generalized amplitude, damping ratio, natural frequency and phase, respectively. 


\section{Introduction}

The application of offshore structures has extended from the oil and gas industry to the offshore wind industry, and it has become critical to assess the structural reliability of offshore structures to prevent unexpected damage/failure [1-4]. From the viewpoint of system control theory, dynamic responses of a structure contain important information about the structure and its excitations simultaneously. In other words, if the important information can be properly extracted from the measured responses, then characteristics of the structure and its excitations can then be further studied by researchers in different areas. This can be achieved by using the signal decomposition method, which can decompose an original signal into a series of components. However, in most engineering issues, the expected components are not easily obtained due to the limitations of the applied algorithm, different natures of the analyzed signals, measured noises, etc. [5].

In recent decades, various signal decomposition methods have been studied, and the Fourier-based method has been widely used for signal decomposition. The Fourier-based method spreads the signal over the space of complete orthogonal sine and cosine functions and achieves the conversion of the signal from time to frequency domain [6]. However, due to the linear and periodic conditions, when the method is applied for measured signals, there will be contradictory phenomena such as false frequencies and energy leakage [7]. To avoid the periodicity hypothesis, Huang et al. [8] proposed the Empirical Mode Decomposition (EMD) based on the concept of instantaneous frequency. One obvious advantage of EMD is that it does not require prior knowledge and can adaptively decompose signals, so this method has been widely used in various fields $[9,10]$. However, when the frequencies of two components are close, EMD will not function well and results will be influenced by mode mixing. Due to these shortcomings, when EMD is used for processing measured signals, results are not ideal.

To solve the problem of mode mixing in EMD, Wu and Huang [11] proposed the Ensemble Empirical Mode Decomposition (EEMD) by adding Gaussian white noise to the original signal so that the blank frequency domain is filled and the screening effect is improved. To date, EMD-based methods have been successfully applied in many aspects, such as mode extraction [12] and biomedical image [13]. In 2014, Dragomiretskiy and Zosso [14] proposed a completely nonrecursive Variational Mode Decomposition (VMD) method by transforming decomposition into a variable decomposition problem and used the alternating direction multiplication method to optimize it. However, the number of extracted modes in VMD needs to be determined before decomposition. If the number of modes is improperly set, the loss or aliasing 
of information in the measured signal may occur, which limits its practicability in processing measured signals. Zhao et al. [15] proposed a quadratic penalty item optimal VMD method based on single-objective salp swarm algorithm to alleviate the mode mixing of complex vibration signals and maintained the signal fidelity for complex vibration signal without prior knowledge. Lian et al. [16] proposed a method that introduced columns of indicators to judge the accuracy of decomposition to automatically determine the mode order. The simulated and measured signals from a hydropower plant were successfully analyzed by this method.

In addition, there exists an earlier method, i.e., the Prony method, which was proposed by the French scientist Gaspard de Prony and is based on a complex exponential model [17]. One can find some application of this method in signal processing [18], dynamic response analysis [19], spectral estimation [20], and so on. However, one significant shortcoming of the traditional Prony method is that it is very sensitive to noise because the process of solving a high-order polynomial is typically an ill-conditioned problem in math. To improve the accuracy of the Prony method when noise is present, there are two types of methods: to obtain better results by optimizing the sampling interval or window size [21], and to filter the time series data before or after using the Prony method [22]. Based on the state-space model, Hu et al. proposed a signal decomposition method called the Prony-SS method [23]. By introducing a first-order matrix difference equation, the ill-conditioned problem of rooting the high-order polynomial equation can be avoided, and the numerical conditionality and robustness are significantly improved. Liu et al. [24] proposed an iterative noise extraction and elimination method for noise contaminated signals, and the interference of high-energy noise on modal parameter identification of offshore wind turbines can be reduced significantly. But it only focused on the elimination of high-energy noise, and did not consider the decomposition of different components in the measured signals.

Based on the concept of one-by-one component extraction, a new signal decomposition method is proposed in this paper. Different from the EMD method in which each component is decomposed one by one according to the frequencies from high to low, only the most significant component is extracted at each decomposition in terms of calculated magnitudes or their power spectral density function, aiming at improving the accuracy of each decomposed component. Because each component can be extracted with an expected accuracy, it is reasonable to expect that the proposed method should have better accuracy in signal decomposition. In the following studies, numerical, experimental and sea-test data are used to investigate the performance of the approach. Specifically, the numerical example is a single degree-of-freedom system 
used to demonstrate and make comparisons with EMD-based methods and the newly developed Prony-SS; the experimental data are from a VIV test by the Norwegian Deepwater Programme (NDP); and the sea-test data are from a fixed platform under wave loadings and from an offshore wind turbine (OWT) in service, collected to study potential applications in engineering.

\section{Preliminaries}

\subsection{The ensemble empirical mode decomposition}

To suppress the mode mixing of EMD, the EEMD was developed by adding Gauss white noise. Steps of the algorithm include the following [25]:

(1) A set of Gaussian white noises $y_{n}(t)$ is added to the original signal $y(t)$, and then an overall signal $\bar{y}(t)$ can be obtained as

$$
\bar{y}(t)=y(t)+\alpha_{n} \cdot y_{n}(t)
$$

where $\alpha_{n}$ is the amplitude coefficient of the white noise. Perform the EMD on Eq. 1, and the signal can be expressed as a sum of Intrinsic Mode Functions (IMFs).

(2) Repeat step (1) by adding different white noise, and take an average of estimated IMFs, as

$$
\overline{c_{i}}=\frac{\sum_{n=1}^{N_{n o i}} c_{i, n}}{N_{n o i}}
$$

where $\overline{c_{i}}$ is the $i$ th IMF, $c_{i, n}$ is the $n$th IMF under $i$ th EMD, and $N_{\text {noi }}$ means the number of noises.

\subsection{The Prony-SS method}

The Prony-SS method, which was recently proposed by Hu et al. [23], which is a technique that uses the state-space model to replace the high order homogenous difference equation, with the capacity of better conditioning and stability. Similar to the Prony's method, the Prony-SS method also includes three steps:

Step 1: Write the discrete signal $y\left(t_{k}\right)$ in a matrix form, as

$$
\left[\begin{array}{cccc}
y_{0} & y_{1} & \cdots & y_{p-1} \\
y_{1} & y_{2} & \cdots & y_{p} \\
\vdots & \vdots & \ddots & \vdots \\
y_{N_{p}-p-1} & y_{N_{p}-p} & \cdots & y_{N_{p}-2}
\end{array}\right]\left[\begin{array}{c}
b_{0} \\
b_{1} \\
\cdots \\
b_{p-1}
\end{array}\right]=\left[\begin{array}{c}
y_{p} \\
y_{p+1} \\
\cdots \\
y_{N_{p}-1}
\end{array}\right]
$$

In the above equation, $p$ represents the number of terms and should be specified in advance; $b_{0}, b_{1}, \cdots, b_{p-1}$ are the determined parameters; $N_{p}$ is the number of sampled points. 
Step 2: Apply the state-space model to calculate roots (denoted as $z_{n}$ ) of the characteristic equation of the $p$ th-order homogeneous linear ordinary differential equation.

Step 3: Use the following equation to estimate amplitudes $\rho_{n}$ and phases $\theta_{n}$, by

$$
\left[\begin{array}{cccc}
z_{1}^{0} & z_{2}^{0} & \cdots & z_{p}^{0} \\
z_{1}^{1} & z_{2}^{1} & \cdots & z_{p}^{1} \\
\vdots & \vdots & \ddots & \vdots \\
z_{1}^{N_{p}-1} & z_{2}^{N_{p}-1} & \cdots & z_{p}^{N_{p}-1}
\end{array}\right]\left\{\begin{array}{c}
\rho_{1} e^{i \theta_{1}} \\
\rho_{2} e^{i \theta_{2}} \\
\vdots \\
\rho_{p} e^{i \theta_{p}}
\end{array}\right\}=\left\{\begin{array}{c}
y_{0} \\
y_{1} \\
\vdots \\
y_{N_{p}-1}
\end{array}\right\}
$$

\section{Signal decomposition based on maximum energy component extraction}

Similar to traditional signal decomposition methods, which usually represent an original signal $f(t)$ by a combination of multiple components, one has

$$
f(t)=\sum_{n=1}^{N_{f}} f_{n}(t)
$$

where $N_{f}$ is the number of components.

\subsection{Representation of structural responses and definition of maximum energy component}

In contrast to most methods, we focused on the issue of decomposing dynamic responses of an undercritically damped structure subject to environmental excitations such as waves, currents, etc., thus, $f_{n}(t)$ can be interpreted as follows

$$
f_{n}(t)=f_{n}^{t}(t)+f_{n}^{s}(t)
$$

where $f_{n}^{t}(t)$ and $f_{n}^{s}(t)$ are the transient and steady-state responses of the structure. Specially, for a single degree of freedom (SDOF) system excited by a harmonic excitation, one can obtain the two terms in Eq. 6 as

$$
f_{n}^{t}(t)=\rho e^{-\xi \omega t} \cos \left(\omega_{d} t+\theta\right)=\rho e^{i \theta} e^{\left(-\xi \omega+i \omega_{d}\right) t}
$$

and

$$
f_{n}^{s}(t)=\bar{\rho} \cos (\bar{\omega} t-\bar{\theta})=\bar{\rho} e^{i \bar{\theta}} e^{i \bar{\omega}_{d} t}
$$

where $\rho, \xi, \omega$ and $\theta$ are the amplitude, damping ratio, natural frequency and phase, respectively, of the transient response; and $\omega_{d}$ is the damped frequency that has a relationship with $\omega$, i.e., $\omega_{d}=\omega \sqrt{1-\xi^{2}}$. Likewise, $\bar{\rho}, \bar{\omega}$ and $\bar{\theta}$ are the corresponding values of the harmonic excitation. 
Thus, Eq. 5 can be written as [26]

$$
f(t)=\sum_{n=1}^{N_{t}} f_{n}^{t}(t)+\sum_{n=1}^{N_{s}} f_{n}^{s}(t)=\sum_{n=1}^{N_{f}} \tilde{\rho}_{n} e^{i \tilde{\theta}_{n}} e^{\left(-\xi_{n} \tilde{\omega}_{n}+i \tilde{\omega}_{n} \sqrt{1-\xi_{n}^{2}}\right) t}
$$

where $N_{t}+N_{s}=N_{f} ; \tilde{\rho}_{n}, \tilde{\theta}_{n}, \tilde{\omega}_{n}$ are generalized representations of the corresponding values of transient or steady-state responses, and $\xi_{n}$ should be zero for steady-state responses.

In this paper, the maximum energy component is just a relative concept and refers to the most significant component in the decomposed signal. A signal represented by Eq. 9 is determined by a zero mean, stationary Gaussian random process, so its energy distribution can be characterized by its power spectral density function $S_{f}(\tilde{\omega})$. For each component, one has

$$
S_{f}(\tilde{\omega})=\frac{1}{2 \pi} \int_{-\infty}^{\infty} R_{f}(t) e^{-i \tilde{\omega} t} d t
$$

where $R_{f}(t)$ is the autocorrelation function of $f(t)$, and it satisfies the relationship of a Fourier transform pair with $S_{f}(\tilde{\omega})$.

Thus, the maximum energy component can be identified by finding its maximum amplitude shown in Eq. 11 as

$$
S_{f}^{m e c}=20 \log _{10}\left(S_{f}(\tilde{\omega})\right)_{\max }
$$

The above equation shows that the maximum energy component can be quantitatively evaluated when the component is extracted, but the remaining question is how to obtain $\tilde{\rho}, \tilde{\theta}, \tilde{\omega}$ and $\xi$ of this component.

\subsection{Extraction of the maximum energy component}

For a sampled signal with time interval $\Delta t$, Eq. 9 can be rewritten by

$$
f_{k}=f\left(t_{k}\right)=\sum_{n=1}^{N_{f}} \tilde{\rho}_{n} e^{i \tilde{\theta}_{n}} e^{\left(-\xi_{n} \tilde{\omega}_{n}+i \tilde{\omega}_{n} \sqrt{1-\xi_{n}^{2}}\right) k \Delta t}=\sum_{n=1}^{N_{f}} \tilde{\rho}_{n} e^{i \tilde{\theta}_{n}} e^{\lambda_{n} k \Delta t}
$$

where $t_{k}=k \Delta t, k=0,1, N_{p}-1$, and $N_{p}$ is the number of sampled signals.

As discussed in reference [23], the signal $f\left(t_{k}\right)$ can be decomposed into a series of components with a better conditioning number using a state-space model. In this paper, a state-space model is employed with the construction of a Hankel matrix,

$$
\mathbf{H}_{f}(k)=\left[\begin{array}{cccc}
f_{k} & f_{k+1} & \cdots & f_{k+N_{c}-1} \\
f_{k+1} & f_{k+2} & \cdots & f_{k+N_{c}} \\
\vdots & \vdots & \ddots & \vdots \\
f_{k+N_{r}-1} & f_{k+N_{r}} & \cdots & f_{k+N_{r}+N_{c}-2}
\end{array}\right]
$$


where $N_{r}$ and $N_{c}$ are the selected number of rows and columns, respectively, and they satisfy the relationship of $N_{r}+N_{c}+1=N_{p}$.

Since the most significant component will be extracted at each time, the model order, here denoted as $o d$, should be equal to 2 in theory. By setting $k=0$ and employing the singular value decomposition, Eq. 13 can be written in the form [27]

$$
\mathbf{H}_{f}(0)=\left[\begin{array}{ll}
\mathbf{U}_{o d}^{f} & \widetilde{\mathbf{U}}_{o d}^{f}
\end{array}\right]\left[\begin{array}{cc}
\mathbf{S}_{o d}^{f} & \mathbf{0} \\
\mathbf{0} & \widetilde{\mathbf{S}}_{o d}^{f}
\end{array}\right]\left[\begin{array}{c}
\left(\mathbf{V}_{o d}^{f}\right)^{T} \\
\left(\widetilde{\mathbf{V}}_{o d}^{f}\right)^{T}
\end{array}\right]=\mathbf{U}_{o d}^{f} \mathbf{S}_{o d}^{f}\left(\mathbf{V}_{o d}^{f}\right)^{T}
$$

where $T$ is a transpose of a vector, $\mathbf{U}_{o d}^{f}$ and $\mathbf{V}_{o d}^{f}$ are singular vectors with a dimension of $N_{r} \times o d$, and $\mathbf{S}_{o d}^{f}$ has a dimension of $o d \times o d$. The singular values $\widetilde{\mathbf{S}}_{o d}^{f}$ are assumed to be zero when the rank of the matrix is exceeded $o d$. In theory, the model order $o d$ is equal to the number of non-zero singular values shown in Eq. 14.

According to the realization theory, one can find a relationship between $\mathbf{H}_{f}(0)$ and $\mathbf{H}_{f}(1)$ by substituting $k=1$ into Eq. 13,

$$
\mathbf{H}_{f}(1)=\mathbf{U}_{o d}^{f} \sqrt{\mathbf{S}_{o d}^{f}} \mathbf{A} \sqrt{\mathbf{S}_{o d}^{f}}\left(\mathbf{V}_{o d}^{f}\right)^{T}
$$

where $\mathbf{A}=e^{\mathbf{F} \Delta t}$, and here $\mathbf{F}$ is a similarity transformation of a coefficient matrix of a converted firstorder matrix differential equation. Following that, the damping ratio $\xi_{n}$ and natural frequency $\tilde{\omega}_{n}$ of this component can be calculated. The, by substituting estimated $\tilde{\omega}_{n}$ and $\xi_{n}$ into Eq. 12, one can easily obtain $\tilde{\rho}_{n}$ and $\tilde{\theta}_{n}$. The detailed procedure can be found in [23].

\subsection{Accuracy evaluation of each extracted component}

During the extraction of each maximum energy component, the caused error can be calculated as well as the accuracy of the following components extraction. To improve the accuracy of each extracted component, a stopping criterion $S C_{s w}$ is defined as

$$
S C_{s w}=\left|\frac{\left|\tilde{S}_{f}^{\text {mec }}\right|-\left|S_{f}^{\text {mec }}\right|}{S_{f}^{\text {mec }}}\right|
$$

where $\tilde{S}_{f}^{m e c}$ and $S_{f}^{m e c}$ are maximum values of the power spectral density function with two different model orders $o d ; \mid$ means the absolute of a number.

When Eq. 16 meets the expected accuracy, the model order is used to extract the component. Here, subscript $l$ is used to denote the number of extracted components, and $\tilde{\omega}_{l}, \tilde{\xi}_{l}, \tilde{\rho}_{l}, \tilde{\theta}_{l}$ can be accordingly determined. Using Eq. 12, this component in the time domain can be constructed as

$$
f_{l}\left(t_{k}\right)=\tilde{\rho}_{l} e^{i \tilde{\theta}_{l}} e^{\lambda_{l} k \Delta t}
$$


Therefore, the remaining part of the original signal $f\left(t_{k}\right)$ can be obtained as

$$
f_{\text {rem }}\left(t_{k}\right)=f\left(t_{k}\right)-\sum_{1}^{l} f_{n}\left(t_{k}\right)
$$

Theoretically, $f_{r e m}\left(t_{k}\right)$ should be equal to zero, but for measured data, it will be approaching zero due to random errors or small inconsistencies. The traditional method for evaluating the decomposed performance can be applied by reconstructing all extracted components for the difference from the original one.

\subsection{Procedure of the proposed method}

Four steps should be included during the implementation of the proposed method, as shown in Fig. 1:

- Step 1- determination of MEC: calculate the autocorrelation function of the original signal $R_{f}(t)$ and then find the maximum value using Eqs. 10 and 11.

- Step 2- extraction of the MEC: substitute the original signal into Eq. 13 by implementing Eq. 15 with $o d=2$, obtain eigenvalues of matrix $\mathbf{A}$, and then $\tilde{\omega}_{n}, \xi_{n}, \tilde{\rho}_{n}$ and $\tilde{\theta}_{n}$ can be calculated, respectively.

- Step 3- accuracy evaluation of the MEC: A stopping criterion $S C_{s w}$ is set using Eq. 16. If $S C_{s w}$ satisfies the expected accuracy, this extracted component can be regarded as the real one. Otherwise, the order $o d$ is increased until the satisfactory $S C_{s w}$ is obtained.

- Step 4- combination for signal reconstruction: replace the original signal with the remaining part using Eq. 18 and repeat Steps 1-3 until each relative MEC is extracted. Then; a reconstructed signal can be obtained by combining all extracted MECs using Eq. 12 .

\section{Numerical demonstration: a single-DOF system subjected to a harmonic loading}

In this example, an SDOF system with $m=10 \mathrm{~kg}, c=2 \mathrm{~N} \mathrm{~s} / \mathrm{m}$ and $k=40 \mathrm{~N} / \mathrm{m}$ is used to demonstrate the validity of the proposed method and provide a detailed numerical procedure. Based on eigen analysis, the natural frequency and damping ratio can be calculated as $0.3183 \mathrm{~Hz}$ and 0.05 , respectively. To obtain the dynamic response of this SDOF system, a harmonic loading $x(t)$ with three components is considered as

$$
x(t)=\sum_{n=1}^{3} B_{n} \cos \left(2 \pi f_{n} t+\phi_{n}\right)
$$




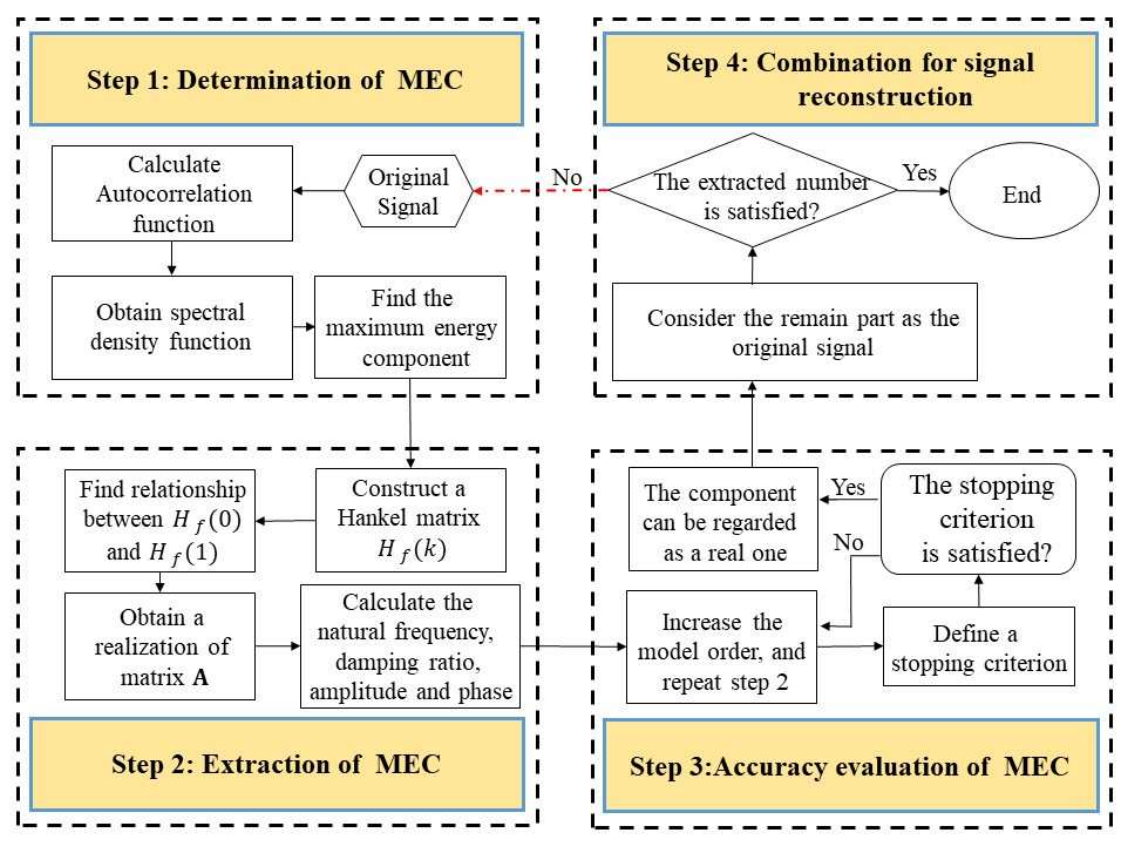

Fig. 1: The flow chart of the proposed method.

where $B_{n}, f_{n}$ and $\phi_{n}$ are the amplitude, frequency and phase of the $n$th component, separately. As an example, these values are set to $B_{n}=[1.2,0.2,0.8], f_{n}=[0.3,0.2,0.8]$ and $\phi_{n}=[-\pi / 4, \pi / 8,0]$, respectively. Fig. 2 shows the simulated loading with $\Delta t=0.1 \mathrm{~s}$.

Two scenarios are taken into account in this example: the first uses the proposed method to decompose the simulated loading, and the second deals with the estimated dynamic response of the system, including making comparisons with traditional methods such as EEMD, VMD and Prony-SS.

\subsection{Scenario 1: decomposition on the simulated loading}

As shown in Eq. 19, the three components are previously known and can be used to investigate the accuracy of the proposed method. As discussed in the procedure of the proposed method, the first step is to determine which component should be extracted first. In this example, based on the autocorrelation function of the original signal $R_{f}(t)$, the maximum value can be calculated using Eq. 11 as $S_{f}^{m e c}=80.7418 \mathrm{~dB}$. In the second step, substituting the sampled data (1500 points in Fig. 2 ) into Eq. 15 with $N_{r}=1500$, one can obtain $N_{c}=1501$. Then, implementing Eq .14, $\mathbf{U}_{o d}^{f}$ and $\mathbf{V}_{o d}^{f}$ have dimensions of $750 \times 2$, and $\mathbf{S}_{o d}^{f}$ is $2 \times 2$, with the preset model order $o d=2$, one can obtain

$$
\mathbf{A}=\left[\begin{array}{cc}
0.9823 & 0.1874 \\
-0.1874 & 0.9823
\end{array}\right]
$$




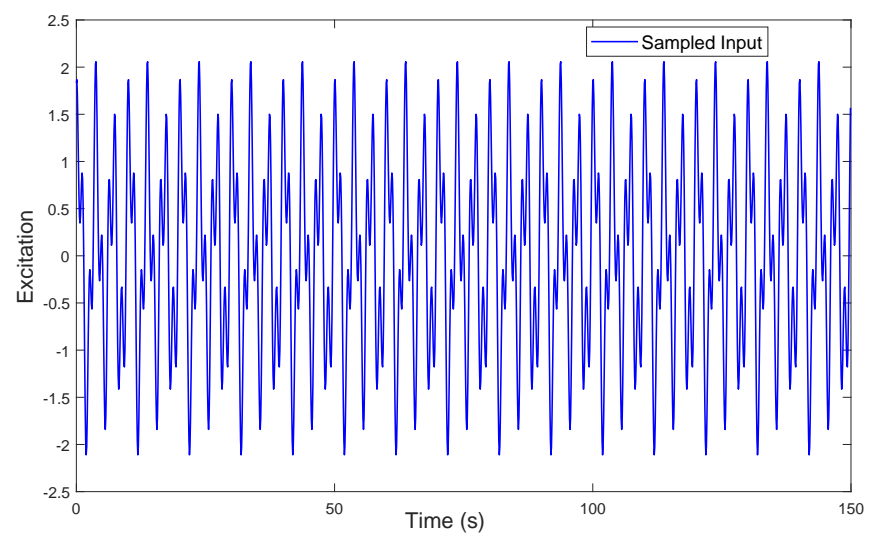

Fig. 2: Simulated loading in terms of Eq. 19.

and $\tilde{\omega}_{n}=1.8848 \mathrm{rad} / \mathrm{s}, \xi_{n}=0.1575 \times 10^{-3}, \tilde{\rho}_{n}=1.2266$ and $\tilde{\theta}_{n}=-0.7747$. Comparing the above values with the true ones, one can find that this component is just the one at $0.3 \mathrm{~Hz}$, and its amplitude is 1.2 , which implies this component is indeed the MEC of the original. Based on step 3, let $o d=4$ and repeat step 2; then, one can obtain $S_{f}^{m e c}=80.8769$, and $S C_{s w}=0.0017$, which means the difference is less than $1 \%$. If this accuracy is acceptable, then one can move to the second MEC extraction, as discussed in step 4. A direct comparison between the three extracted components and their true ones is shown in Fig. 3, and one can find perfect consistency between them, which implies good accuracy of signal decomposition using the proposed method.

\subsection{Scenario 2: decomposition on the estimated dynamic response}

In practice, structural response contains not only information of excitations, but also has a direct reflection of stiffness, mass and damping. Therefore, if the loading shown in Fig. 2 is imposed on the system, an analytical response can be obtained because of the harmonic characteristic of this loading. In addition, a much more general case is considered in which this system has initial conditions, i.e., initial displacement and velocity are randomly set to $0.5 \mathrm{~m}$ and $0.2 \mathrm{~m} / \mathrm{s}$, respectively. Fig. 4 shows the estimated dynamic response with a time duration of 3000 points, which will be used as the original signal for decomposition.

Based on the same procedure yielding Fig. 3, one can decompose Fig. 4 into a series of components. One should note that the only difference is that the extraction number $N_{f}$ is 4 , and $S C_{s w}=1 \times 10^{-6}$ is used to ensure that each MEC can be extracted with sufficient accuracy. For example, during the extraction 

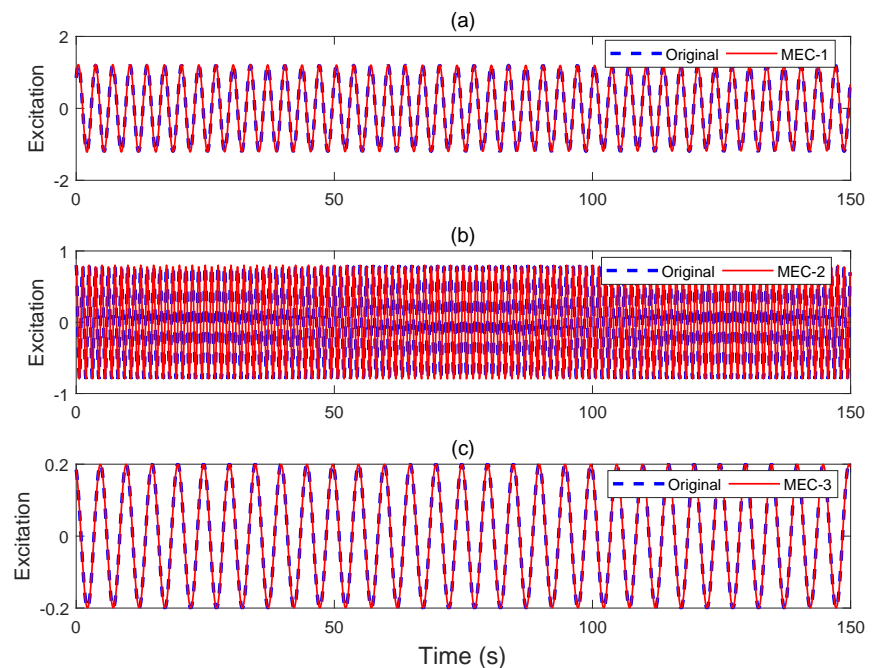

Fig. 3: Comparison between extracted components by the proposed method and true ones.

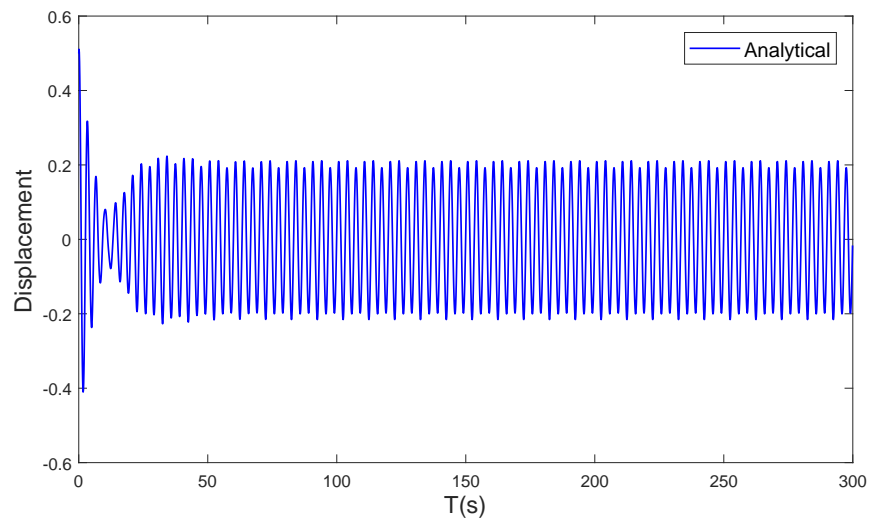

Fig. 4: Analytical response of the system excited by the simulated loading. 

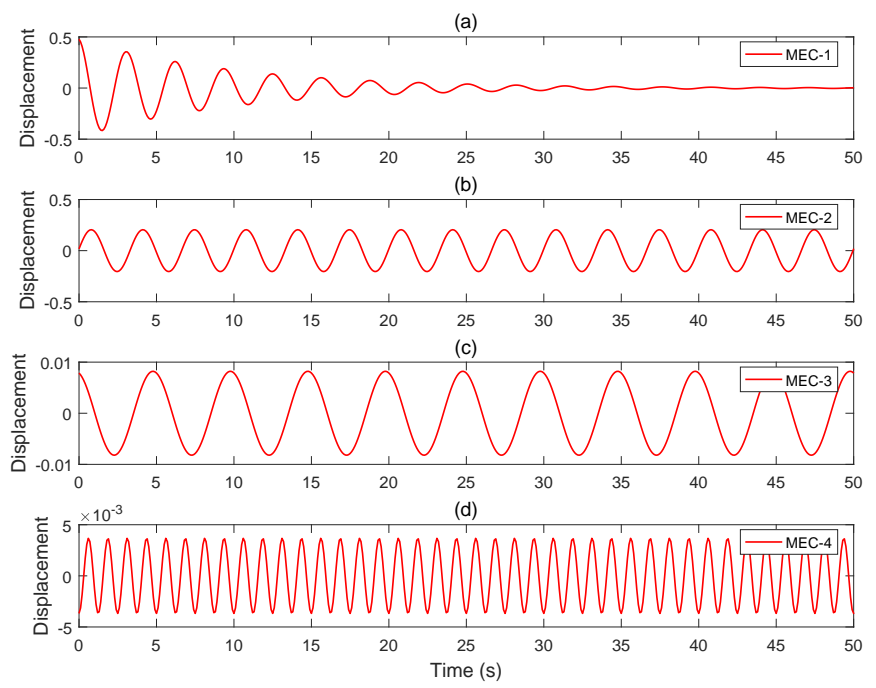

Fig. 5: Four extracted MECs by implementing the proposed method.

of the first MEC, $S C_{s w}$ reached $6.8740 \times 10^{-14}$ when model order is $o d=6$, and this component is shown in Fig. 5 (a), with $\xi=0.0502$ and $\omega=0.3183 \mathrm{~Hz}$, which shows very good estimations of the true values of this system. Shown in Fig. 5 (b)-(d) are the other three extracted components; one can clearly find that their amplitudes are in a descending sequence, and the amplitude of the last one is less than $1 \times 10^{-3}$, which implies that $N_{f}=4$ is suitable.

\subsection{Comparison with traditional signal decomposition methods}

To show the capability of the proposed method, we selected three decomposition methods that have been widely used in recent years to make a comparison, including EEMD, VMD and Prony-SS. EEMD has unique advantages in dealing with nonlinear signals. It is an empirical decomposition method with advantages including adaptive filtering and does not require any transformation. Using white noise, the mode-mixing issue of EMD has been overcome to some extent. Based on the concept of IMF, VMD establishes a clear variational model. It has the anti-noise advantage of a Wiener filter and can balance the backward error caused by non-recursive screening. Prony-SS is a recently developed method where the signal is decomposed into the form of a complex exponential function that avoids the periodic hypothesis of the traditional Fourier transform. By introducing the first-order differential equation, the ill-conditioned problem of the Prony method is overcome, which leads to high precision.

Here, we compare the decomposition results of the second component (shown in Fig. 5), i.e., the com- 


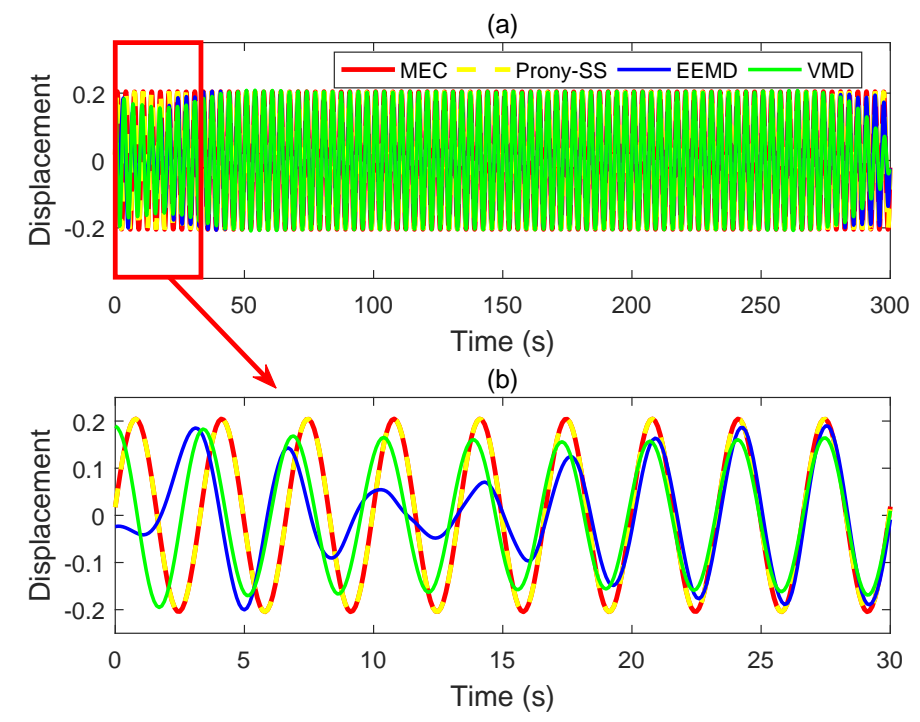

Fig. 6: Comparison of components with $0.3 \mathrm{~Hz}$ extracted by the four methods.

ponent with a frequency of $0.3 \mathrm{~Hz}$. First, with a ratio of the standard deviation of the added noise set to 0.2 , the EEMD method is used to decompose the signal, and 11 components are obtained. The decomposed component with $0.3 \mathrm{~Hz}$ is illustrated in Fig. 6 as the blue line. It can be seen that the components at the endpoint are not stable due to the endpoint effect of EEMD, and it tends to be stable over time. Then, VMD is performed to decompose the signal, and since the dynamic response has four components, the number of modes is set to be 4. As shown with the green line, similar to EEMD, VMD also has the problem of the end-point effect. With a mode order of 8, the result of the Prony-SS is shown as the yellow line. Additionally, the result by the MEC is shown as the red line. All four methods can effectively separate the component with $0.3 \mathrm{~Hz}$. However, due to the influence of the end-point effect, the results of EEMD and VMD are worse than Prony-SS and the MEC. Moreover, the components decomposed by EEMD and VMD are smaller than those by Prony-SS and the MEC, which indicates that Prony-SS and the MEC decompose the signal more thoroughly. For further comparison of Prony-SS and the MEC, the residues after decomposition are shown in Fig. 7. We can see that the residue of the proposed method is smaller, which means the precision of the MEC is higher than Prony-SS. 


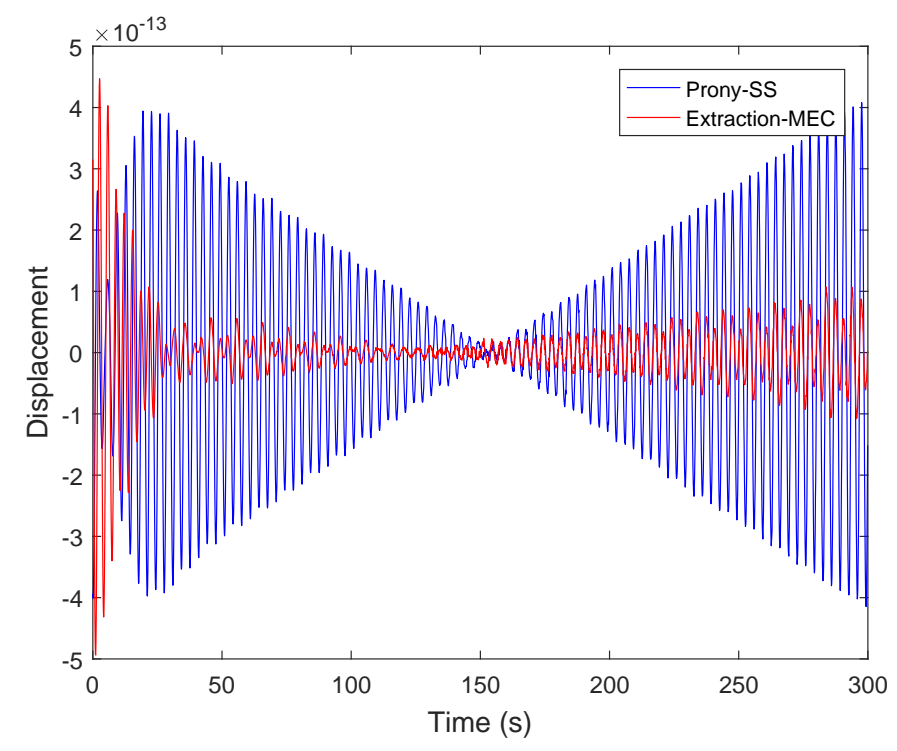

Fig. 7: Comparison of residues by Prony-SS and the MEC.

\section{Experimental data: VIV data from the Norwegian Deepwater Programme}

In this example, the experimental data derived from a Riser High Mode Vortex-Induced Vibration (VIV) test sponsored by the Norwegian Deepwater Programme (NDP) is analyzed. The model is made of composite fibers with an $L / D$ of 1400 , and the dimensions are $L=38 \mathrm{~m}$ and $D=0.0027 \mathrm{~m}$. In each test, the model riser was pulled horizontally at different speeds. When the ends of the riser model were pulled at the same speed, a uniform flow profile was produced; when the model riser was fixed at one end and the other end was pulled, a linear shear flow profile was produced. The details of the experiment are recorded in $[28,29]$.

During the experiment, the vibration is mainly produced by the coupling motion in the crossflow and inline directions, and there are other interferences such as noises. Therefore, the measurement signal is mixed with many frequency components. Through signal decomposition, different frequencies can be identified. At the same time, the frequency of the inline response is twice the frequency of the crossflow motion [30]. These details can also help us to demonstrate the correctness of the signal decomposition. Here, we selected the acceleration data as $\Delta / L=0.1314$ in the inline direction for analysis. The corresponding towing speed is $0.7 \mathrm{~m} / \mathrm{s}$. The chosen signal with a duration of $10 \mathrm{~s}$ in the time domain and frequency domain are illustrated in Fig. 8.

When implementing EEMD, which is an adaptive decomposition method, a total of 14 IMFs was ob- 
(a)

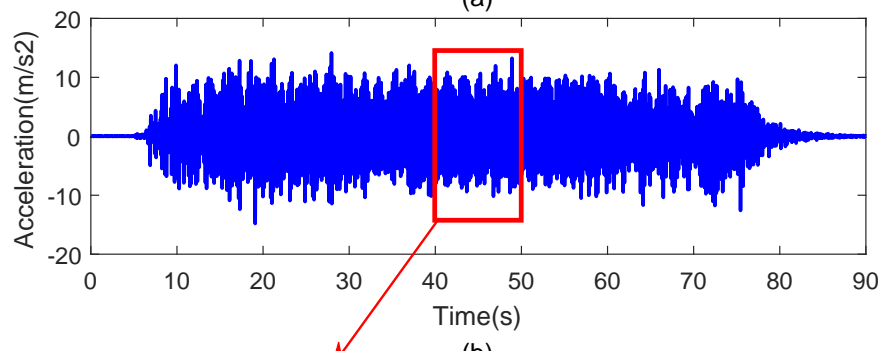

(b)

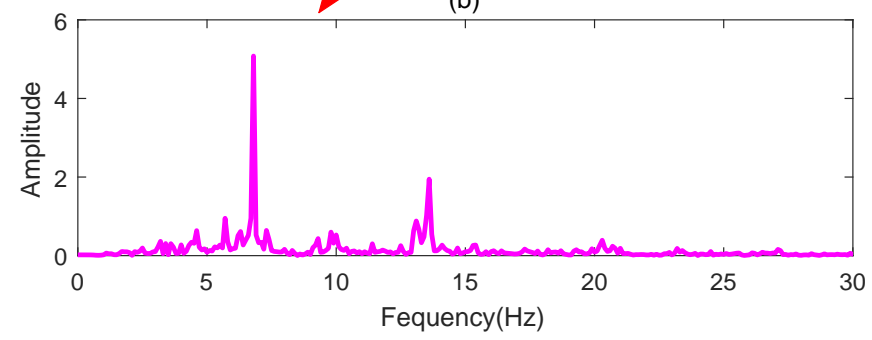

Fig. 8: The waveform of the signal and the chosen signal of $10 \mathrm{~s}$ in the frequency domain.

tained, as shown in Fig. 9. Because the first decomposed component is noise, we only list the second IMF to the sixth IMF. From the results, we can see that the signal is decomposed from high frequency to low frequency. However, due to the effect of mode-mixing, the decomposition is not accurate. For example, the component with $6.8 \mathrm{~Hz}$ not only exists in IMF 2, but can also be seen in IMF 3 and IMF 4; and the component with $13.6 \mathrm{~Hz}$ exists in IMF 2 and IMF 3 simultaneously. As mentioned in Ref. [31], the problem of mode mixing has become one of the greatest drawbacks of EMD. Although the addition of white noise has greatly improved the problem of mode mixing in EMD, for signals with very close frequencies, the performance of EEMD is not satisfactory, and the components still cannot be successfully separated. At the same time, because of noise, there are many meaningless low-frequency components, and this will lead to serious over-binding.

It can be seen in Fig. 8 that the energy of two components is greater than the other components. Therefore, when using VMD, the mode is set to 2, and the results are shown in Fig. 10. From Fig. 10 (b), we can see the first mode corresponds to the cross-flow response; the second mode corresponds to the response in the inline direction. However, due to the lower decomposition mode, each mode contains many frequencies. These results are due to a priori nature of VMD, that is, before using VMD to decompose, the number of decomposed modes is set artificially. If the set order of decomposition is not correct, the result will be incorrect. Therefore, the choice of decomposed mode is crucial when using VMD to decompose the 

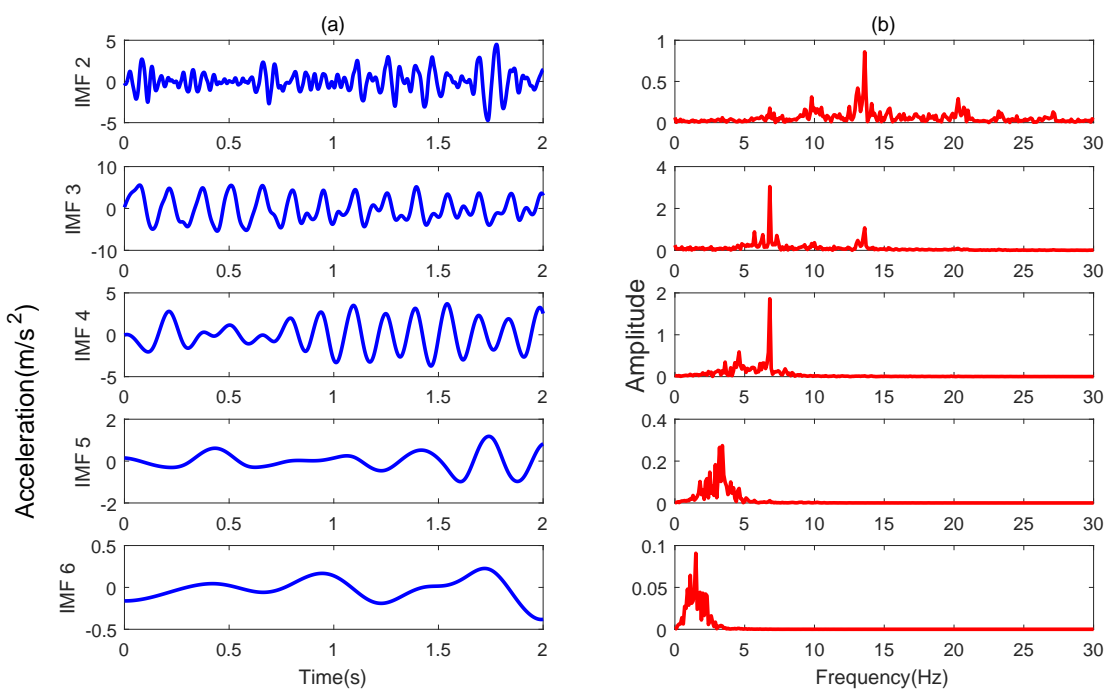

Fig. 9: The decomposition results of EEMD in (a) time domain and (b) frequency domain.

signal. However, when processing measured data, due to the influence of complex environment, the signal is polluted by noise, and it is difficult to determine the number of meaningful modes buried in the signal. Thus, due to blindness and randomness, the reasonable selection of parameters and the problem of underor over-decomposition of VMD limit its application in engineering.

When implement Prony-SS, the model order is set to 4, and the decomposed two components are illustrated in Fig. 11 as the blue line. When the extraction number $N_{f}=2$ and $S C_{s w}=1 \times 10^{-6}$, the results obtained by using the MEC are also shown in Fig. 11 as the red lines. Fig. 11 (b) shows the extracted components in the frequency domain. We can see that both methods can successfully decompose the two main components. In the first component, there are very small differences, and the results from the two methods show good consistency. However, the amplitude of the second component extracted by the MEC is larger than that obtained by Prony-SS. This means that after the decomposition, the MEC has smaller residual and is thus more accurate than Prony-SS.

\section{Field test study:}

To study the performance of the proposed method in dealing with actual signals of offshore structures, the measured data of two typical offshore structures were used: one from the WZ11-4D fixed platform under wave excitation, and the other from an operational offshore wind turbine. Since Prony-SS and the proposed method are superior to EEMD and VMD in the analysis of VIV data, we only used the results of 
(a)
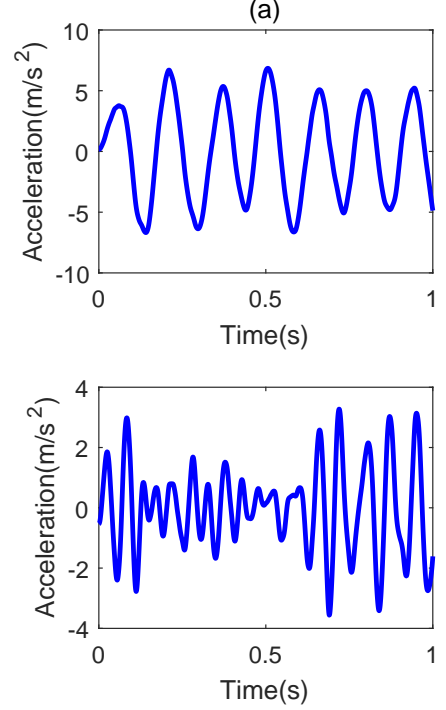

(b)
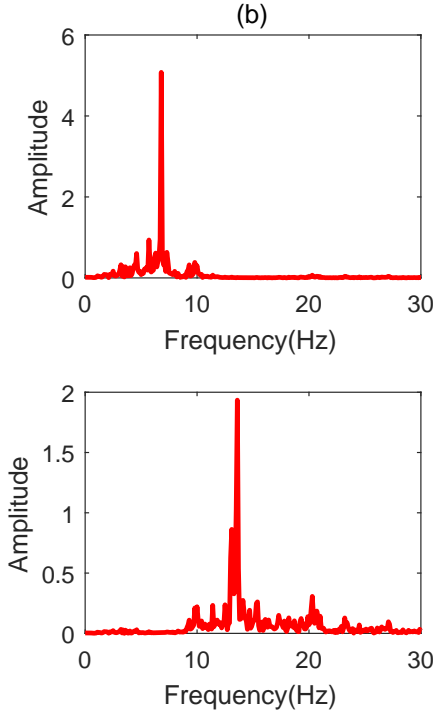

Fig. 10: The results of decomposition by the VMD in (a) time domain and (b) frequency domain.
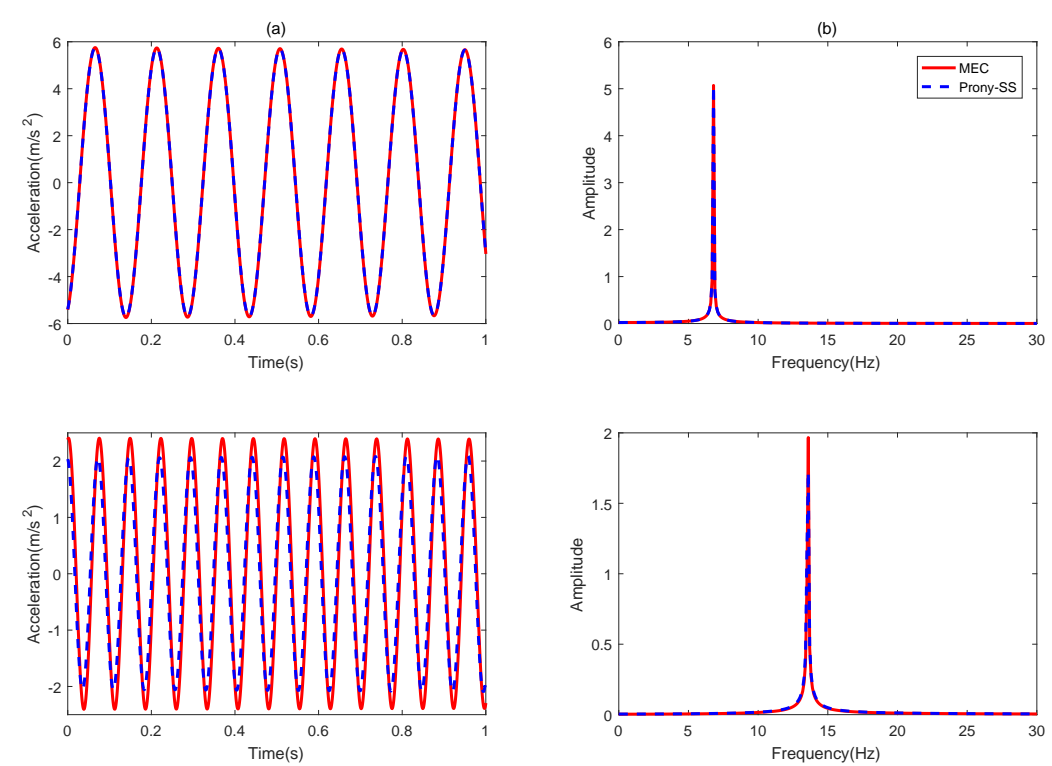

Fig. 11: The decomposition results of Prony-SS and the MEC in (a) time domain and (b) frequency domain. 

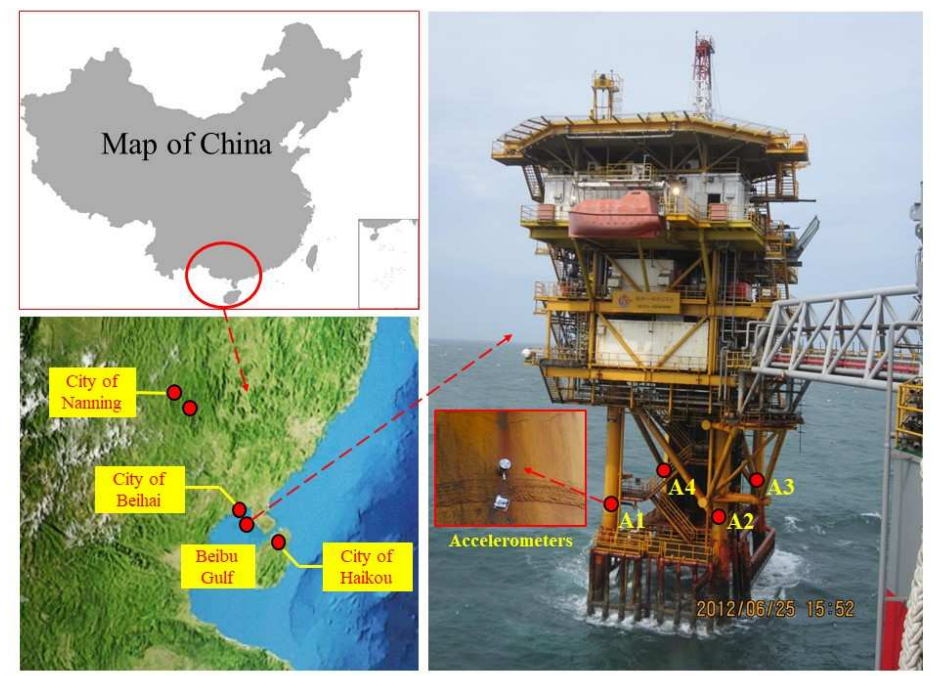

Fig. 12: The tested platform in the Beibu Gulf of China.

Prony-SS for comparison in the following studies.

\subsection{Platform under wave excitation}

In this case, a fixed platform located in the southwest of Weizhou Island in the North China Sea was used for measurement. The platform is a jacked structure with four legs and weighs 805.55 tons. The design life of the platform is 20 years. The main pile of the platform is $4 \times \phi 48^{\prime \prime}, 67 \mathrm{~m}$ into the mud and weighs 710 tons; the pipe group is $4 \times \phi 48^{\prime \prime}, 67 \mathrm{~m}$ into the mud and weighs 504 tons. The area of the deck is $890 \mathrm{~m}^{2}$, and its weight is 486 tons. The weight of the equipment on the deck is 121.6 tons. Taking the static water level as a reference point, the deck is divided into three layers, and the elevation of each layer is $20.84 \mathrm{~m}, 25.84 \mathrm{~m}$ and $37.0 \mathrm{~m}$, respectively. The plane size of the structure at the working point is 18.288 $\mathrm{m}$ long and $9.144 \mathrm{~m}$ wide. Acceleration sensors are arranged at the joints of the four main pile legs $(+7.5$ m). Fig. 12 shows the geographic location, measuring point arrangement and tri-axial accelerometers of the target platform. In each test, the acceleration signals were recorded for 4 hours with a sampling rate of 500 Hz. In this case, the measured data at A-1 were selected for analysis, and the waveform of the measured data is shown in Fig. 13 (a). Data of $50 \mathrm{~s}$ (from $1700 \mathrm{~s}$ to $1750 \mathrm{~s}$ ) are selected for decomposition, and the PSD spectrum of the chosen signal is shown in Fig. 13 (b).

Because of the good performance of the Prony-SS on signal decomposition, one may expect that this method can also give good results. As discussed in Eq. 3, the number of terms should be selected in advance and it has a direct influence on the accuracy of signal decomposition, so the order was set to increase from 

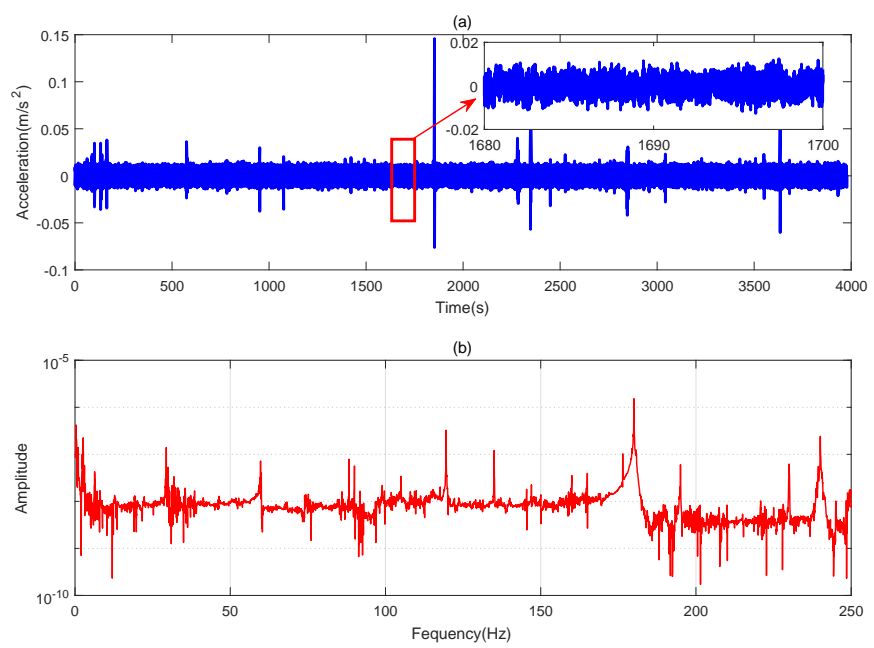

Fig. 13: The measured signal in (a) time domain and (b) frequency domain.

100 to 3000 with an interval of 500. The decomposition result was quantitatively evaluated by introducing a correlation function defined as [32]

$$
\rho_{x_{r} x_{o}}=\frac{\operatorname{cov}\left(x_{r}, x_{o}\right)}{\sigma_{x_{r}} \sigma_{x_{o}}}=\frac{E\left(x_{r} x_{o}\right)-E\left(x_{r}\right) E\left(x_{o}\right)}{\sqrt{E\left(x_{r}^{2}\right)-E\left(x_{r}\right)^{2}} \sqrt{E\left(x_{o}^{2}\right)-E\left(x_{o}\right)^{2}}}
$$

where $x_{r}$ represents the reconstructed component, $x_{o}$ represents the original component, and $E$ represents the expectation. Using Eq. 21, the correlation coefficient $\rho_{i}$ between the reconstructed and the measured signal can be calculated, and the corresponding results of $\rho_{i}$ are illustrated in Fig. 14 (b) as the blue line. As the order increases, the correlation coefficient increases, but when the order exceeds 2300 , the reconstructed results completely deviates from the original signal, as indicated by the nearly zero $\rho_{i}$. Fig. 14 (a) shows the result of reconstruction when the order is 3000 and the blue line denotes the measured signal. We can see that at the beginning, the result of reconstruction is accurate, but as time increases, the error of reconstruction becomes larger. This is because of the utilization of the state-space model, whose input should be an impulse function, while the input of these measured data are continuous environmental loads from waves, currents and so on.

When using the MEC method, to make a comparison with Prony-SS, the number of extractions was set from 50 to 1500 (corresponding to the order from 1000 to 3000 in Prony-SS) and the stopping criterion is set to $S C_{s w}=1 \times 10^{-6}$. The correlation function is also used to quantify the reconstruction error as the red line in Fig. 14 (b). It can be seen that when the extracted number in the MEC corresponds to the order, the correlation coefficient of the MEC is larger than the results of Prony-SS. This means that under 

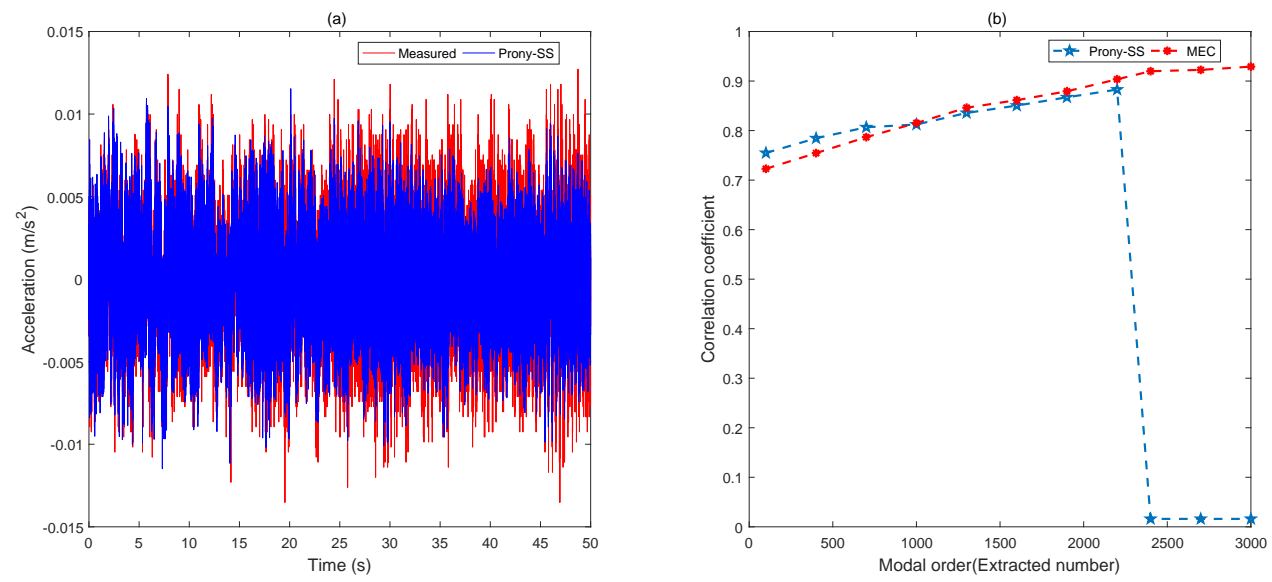

Fig. 14: (a) The reconstructed signal by Prony-SS; (b) The correlation coefficient with different order.

the same conditions, the decomposition accuracy of the MEC is higher than Prony-SS. At the same time, it can be seen that when the number of extractions is increased to a large value, the result of the MEC is still accurate, and there is no problem of divergence. Fig. 15 plots the reconstructed result of the MEC versus the measured signal when the number of extractions is set to 3000 . It can be seen that the reconstructed result is consistent with the measured signal, and the problem of inaccurate decomposition over time in Prony-SS has been overcome.

\subsection{An offshore wind turbine in service}

The measured OWT is located in the region of the Yellow Sea near Jiangsu Province, China. The height of the turbine is $86 \mathrm{~m}$, and the foundation is a single pile structure. During the test, the dynamic signal of the structure was acquired using an IMC collector and two tri-axial accelerometers, which were arranged inside the tower. The first three modal frequencies of the operational OWT are $0.3097 \mathrm{~Hz}, 1.7762 \mathrm{~Hz}$, and 3.9910 $\mathrm{Hz}$ with their corresponding damping ratios of $0.0269,0.0661$, and 0.0277 [33]. In this measurement, a signal with the duration of 1 hour was recorded under each operating condition and the sampling frequency was $100 \mathrm{~Hz}$. Fig. 16 shows a prototype of the measured offshore wind turbine, the arrangement of the measuring points, the tri-axial accelerometers and the signal acquisition system arranged on the OWT.

From these assembled accelerometers, one can obtain the dynamic responses of the offshore wind turbine, and as shown in Fig. 17, which shows the measured time series and its PSD spectrum. From the PSD spectrum (Fig. 17 (b)), we can see that in the measured response, there are not only the structural mode of the offshore wind turbine but also the harmonic components caused by the rotation of the turbine blades 

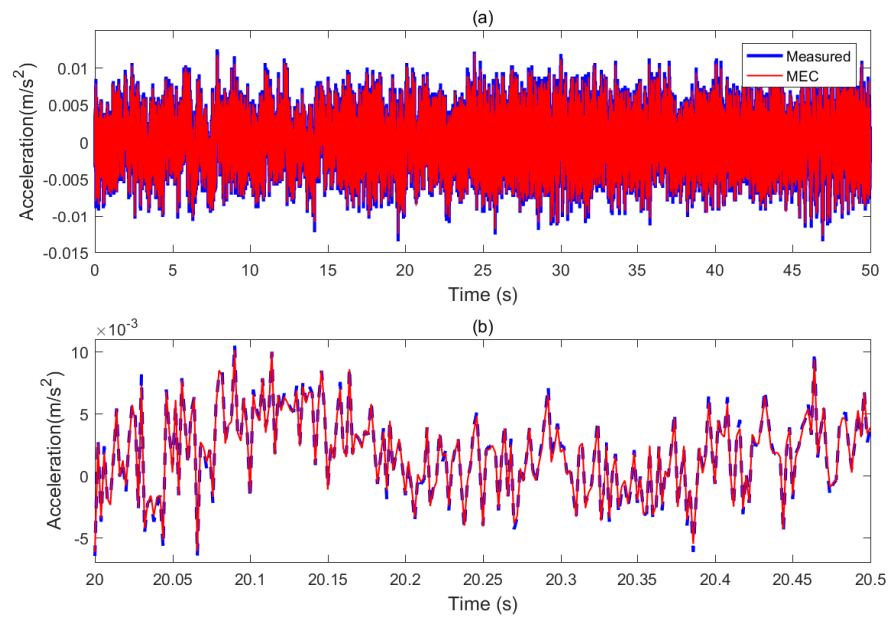

Fig. 15: The reconstructed signal by the MEC.

[24]. To further understand the components in the response, the stochastic subspace identification (SSI) is used to identify the modal information as shown in Fig. 18. The results are consistent with the frequencies of the first six peaks in the spectrum, which are 0.3988, 0.7985, 1.6000, 1.1978, 2.0012 and $2.3880 \mathrm{~Hz}$. In the subsequent analysis, the signal from $900 \mathrm{~s}$ to $1000 \mathrm{~s}$ was chosen to be decomposed.

By setting the extraction number $N_{f}$ to 50 , and $S C_{s w}=1 \times 10^{-6}$, Fig. 19 shows the six components corresponding to the six peaks decomposed from the measured signal. In Fig. 19, the red lines represent the decomposed components in the frequency domain and the blue lines are the decomposed components in the time domain (in the time domain, the horizontal axis is time (s) and the vertical axis is acceleration $\left.\left(\mathrm{m} / \mathrm{s}^{2}\right)\right)$. The frequencies of the six decomposed components are $0.3990,0.7982,1.1933,1.5994,1.9957$ and $2.3893 \mathrm{~Hz}$, which are almost identical to the results of SSI. When using the MCE, each component can be decomposed, which proves the superiority and potential value in processing the measured signals in real-world engineering problems.

\section{Conclusions}

In this paper, a novel signal decomposition method based on the concept of one-by-one component extraction has been proposed. By representing the components in a complex exponential form, Maximum Energy Components (MECs) corresponding to the structure and excitations have been successfully separated. By introducing a controlled accuracy criterion, each component has been precisely extracted. Numerical results show that the proposed method has the similar accuracy as Prony-SS in decomposing the synthe- 

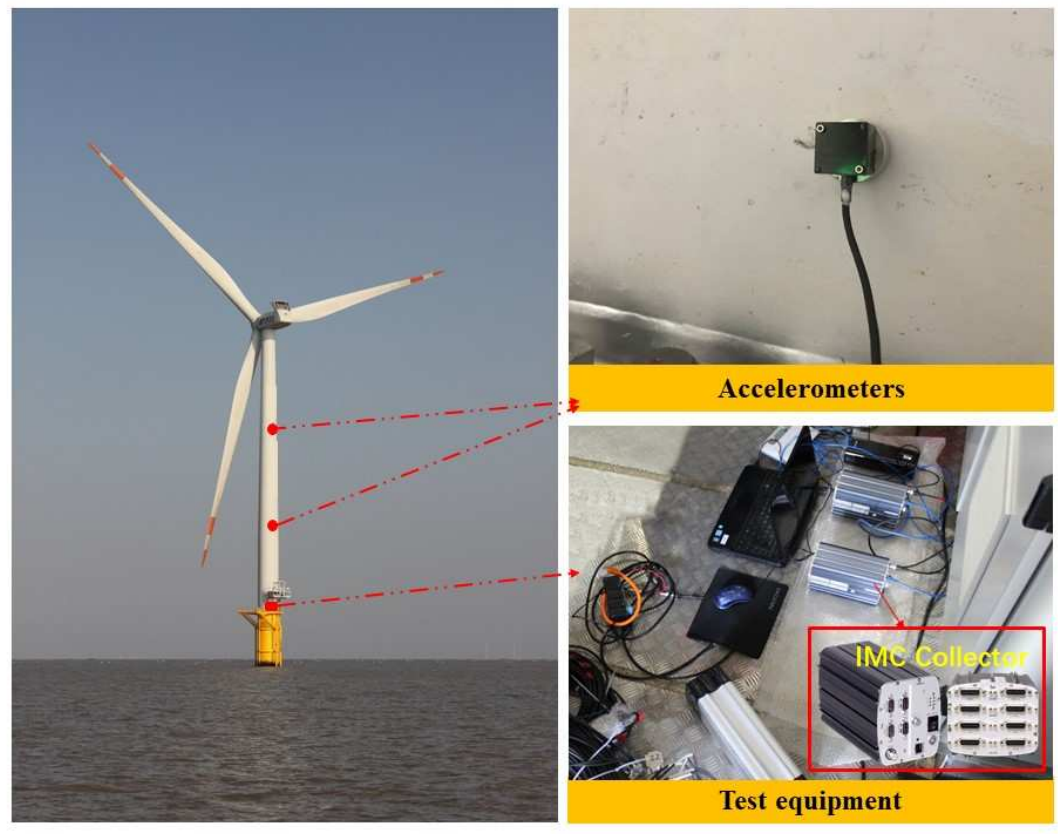

Fig. 16: The tested turbine and the test equipment.
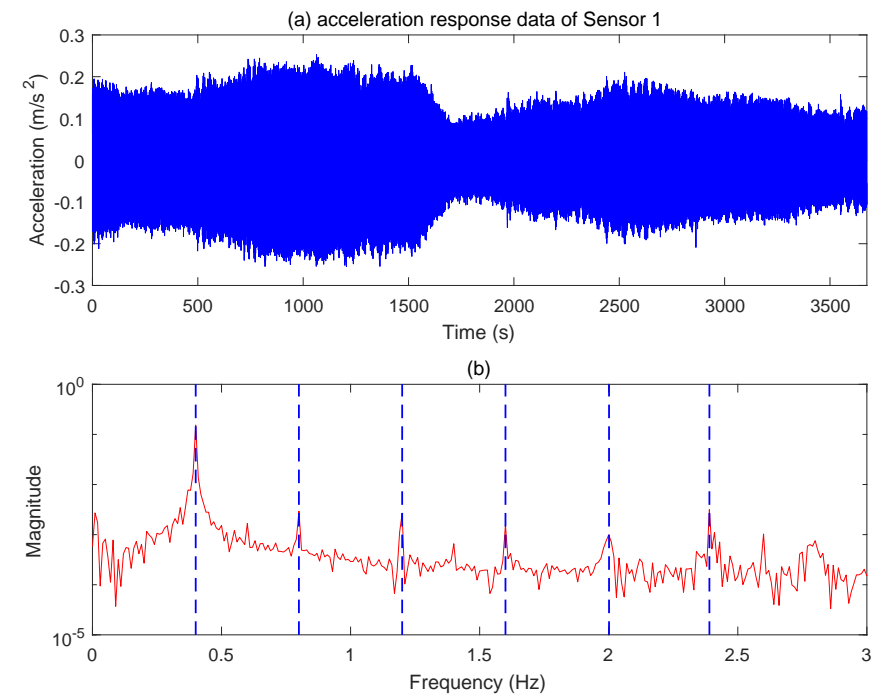

Fig. 17: The measured signal and its PSD spectrum. 


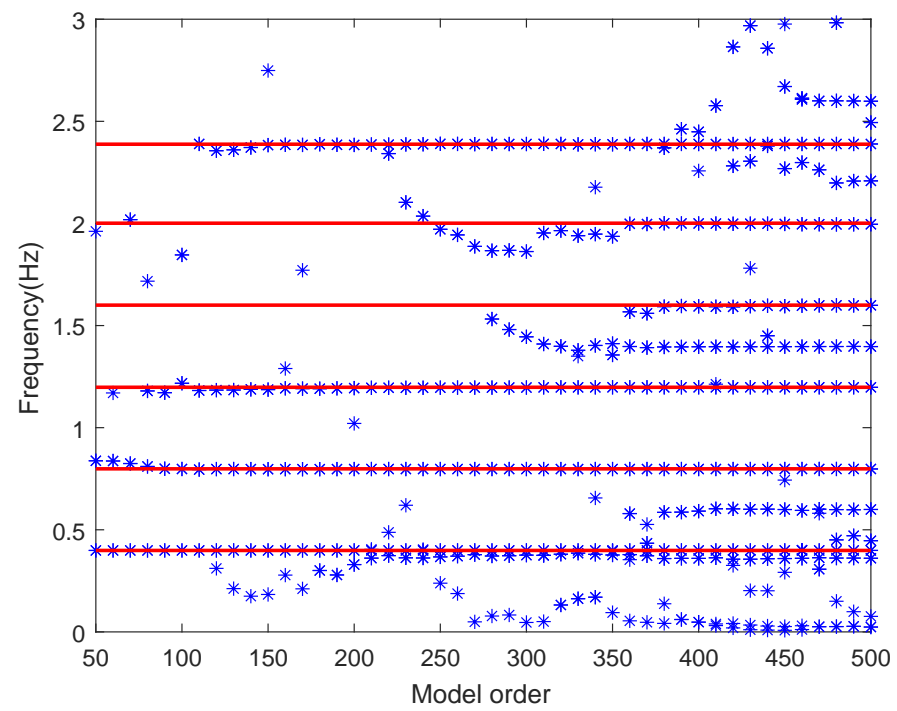

Fig. 18: Identified frequencies by SSI.
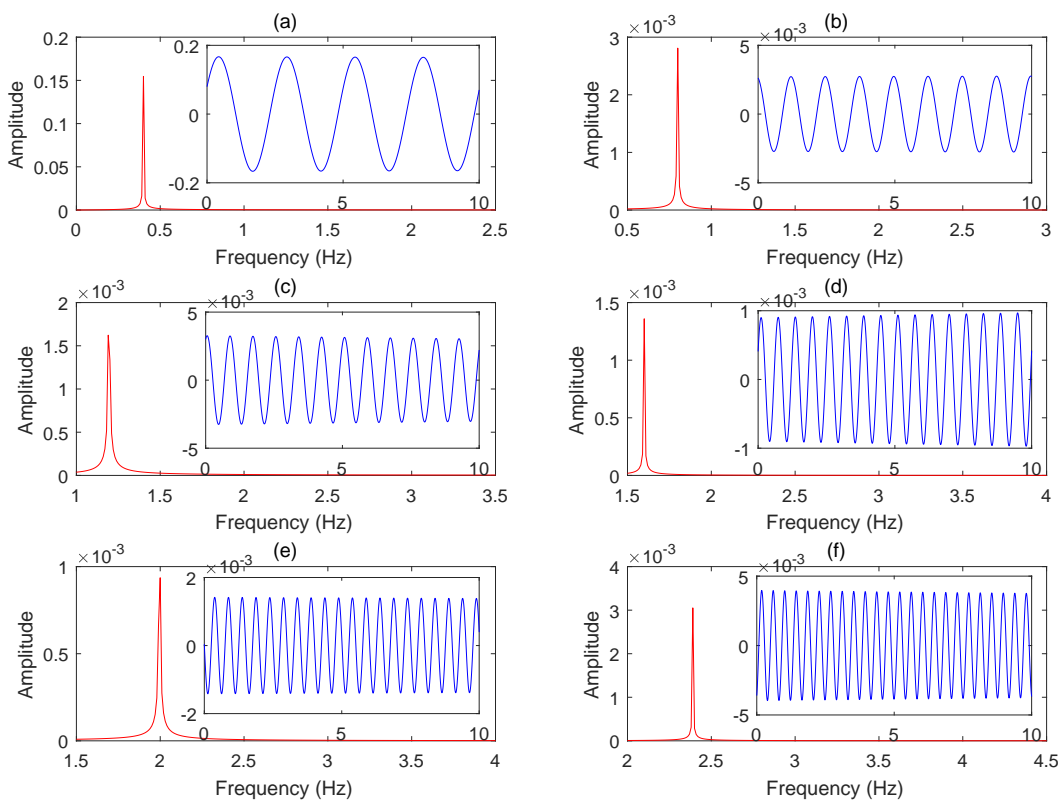

Fig. 19: Six decomposed components by the MEC. 
sized four components and outperforms EEMD and VMD. Furthermore, the stability and accuracy of the proposed approach have also been demonstrated by examining the experimental and sea-test data. It is noted that results by Prony-SS show the divergence and cannot provide the good decomposition of signals when a large number of order is adapted, while the developed method shows a more stable capability of signal decomposition and reconstruction with a high level of accuracy.

\section{Acknowledgements}

The authors acknowledge the financial support of the National Natural Science Foundation of China (grant no. U1806229), the Taishan Scholars Program of Shandong Province and the generous permission by the Norwegian Deepwater Program authors to use their experimental data.

\section{Reference}

[1] Hamid A, Masoud AN. Effects of geometrical parameters on the degree of bending (DoB) in two-planar tubular DT-joints of offshore jacket structures subjected to axial and bending loads. Mar Struct 2019;64:229-245.

[2] Hamid A, Esmaeil Z. Degree of bending (DoB) in offshore tubular KT-joints under the axial, in-plane bending (IPB), and out-of-plane bending (OPB) loads. Appl Ocean Res 2020;95:102015.

[3] Hamid A, Esmaeil Z. The effect of multi-planarity on the SCFs in offshore tubular KT-joints subjected to in-plane and out-of-plane bending loads. Thin Wall Struct 2016;106:148-165.

[4] Moises J-M. Fatigue of offshore structures: A review of statistical fatigue damage assessment for stochastic loadings. Int $\mathbf{J}$ Fatigue 2020;132:105127.

[5] Dong XF, Lian JJ, Wang HJ. Vibration source identification of offshore wind turbine structure based on optimized spectral kurtosis and ensemble empirical mode decomposition. Ocean Eng 2019;172:199-212.

[6] Brigham EO. The fast Fourier transform and its applications. New Jersey: Prentice Hall 1988.

[7] Liu FS, Chen JF, Qin HD. Frequency response estimation of floating structures by representation of retardation functions with complex exponentials. Mar Struct 2017;54:144-166.

[8] Huang NE, Shen Z, Long SR, Wu MLC, Shih HH, Zheng QA, Yen N-C, Tung CC, Liu HH. The Empirical Mode Decomposition and the Hilbert Spectrum for Nonlinear and Non-Stationary Time Series Analysis. Proc R Soc A-Math Phys Eng Sci 1998;454:903-995.

[9] Li C, Wang XL, Tao ZY, Wang QF, Du SP. Extraction of time varying information from noisy signals: An approach based on the empirical mode decomposition. Mech Syst Signal Process 2011;25:812-820.

[10] Duan WY, Han Y, Huang LM, Zhao BB, Wang MH. A hybrid EMD-SVR model for the short-term prediction of significant wave height. Ocean Eng 2016;124:54-73.

[11] Wu ZH, Huang NE. Ensemble empirical mode decomposition: a noise-assisted data analysis method. Advances in Adaptive Data Analysis 2009;1(1):1-41. 
[12] Zhang B, Jiang ZN, Feng K. Research on variational mode decomposition in rolling bearings fault diagnosis of the multistage centrifugal pump. Mech Syst Signal Process 2017;93:460-493.

[13] Lahmiri S, Boukadoum M. Biomedical image denoising using variational mode decomposition. IEEE BioCAS 2014:340343.

[14] Konstantin D, Zosso D. Variational Mode Decomposition. IEEE Trans Signal Process 2014;62(3):531-544.

[15] Zhao XL, Wu PF, Yin XX. A quadratic penalty item optimal variational mode decomposition method based on singleobjective salp swarm algorithm. Mech Syst Signal Process 2020;138:106567.

[16] Lian JJ, Liu Z, Wang HJ, Dong XF. Adaptive variational mode decomposition method for signal processing based on mode characteristic. Mech Syst Signal Process. 2018;107:53-77.

[17] Prony GCFMRD. Essai experimental et analytique: Sur les lois de la dilatabilite des fluids elastiques et sur celles de la force expansive de la vapeur deleau et de la vapeur de lalkool, a differentes temperatures (in French). Journal de l'Ecole Polytechnique 1795;1(2):24-76.

[18] Zhao S, Loparo KA. Forward and backward extended Prony method for complex exponential signals with/without additive noise. Digit Signal Process 2019;86:42-54.

[19] Liu FS, Fu Q, Tian Z. A Laplace-domain method for motion response estimation of floating structures based on a combination of generalised transfer function and partial fraction. Ships Offshore Struc 2019;DOI:10.1080/17445302.2019.1687145.

[20] Luo R, Lv HJ, Liu HQ. Development of Prony series models based on continuous relaxation spectrums for relaxation moduli determined using creep tests. Constr Build Mater 2018;168:758-770.

[21] Peng JCH, Nair NKC. Adaptive sampling scheme for monitoring oscillations using Prony analysis. IET Gener Transm Distrib 2009;3:1052-1060.

[22] Tripath P, Srivastava SC, Singh SN. An improved Prony method for identifying low frequency oscillations using synchrophasor measurements. IEEE ICPES 2010:1-5.

[23] Hu S-LJ, Yang WL, Li HJ. Signal decomposition and reconstruction using complex exponential models. Mech Syst Signal Process 2013;40:421-438.

[24] Liu FS, Gao SJ, Han HW, Tian Z, Liu P. Interference reduction of high-energy noise for modal parameter identification of offshore wind turbines based on iterative signal extraction. Ocean Eng 2019;183:382-383.

[25] Wang WY, Chen QJ, Yan DL, Geng DZ. A novel comprehensive evaluation method of the draft tube pressure pulsation of Francis turbine based on EEMD and information entropy. Mech Syst Signal Process 2019;116:772-786.

[26] Tian Z, Liu FS, Zhou L, Yuan CF. Fluid-structure interaction analysis of offshore structures based on separation of transferred responses. Ocean Eng 2020;195:106598

[27] Golub GH, Loan CFV. Matrix Comptatuons, 3rd edition. Johns Hoppkins University Press, Baltimore, MD, 1996.

[28] Braaten H, Lie H. NDP riser high mode VIV tests main report. Main report number 512394.00.01. Norwegian Marine Technology Research Institute, 2004.

[29] Trim AD, Braaten H, Lie H, Tognarelli MA. Experimental investigation of vortex induced vibration of long marine risers. J Fluids Struct 2005;21:335-361.

[30] Vandiver JK, Ma LX, Rao ZB. Revealing the effects of damping on the flow-induced vibration of flexible cylinders. J Sound Vibr 2018;433:29-54.

[31] Xu GL, Wang XX, Xu XG, Zhou LJ. Improved EMD for the analysis of FM signals. Mech Syst Signal Process 2012;33:181- 
196.

[32] Martin P, Alaa EM. Long-term correlation structure of wave loads using simulation. Mar Struct 2011;24(2):97-116.

[33] Zhou L, Li Y, Liu FS, Jiang ZQ, Yu QX, Liu LJ. Investigation of dynamic characteristics of a monopile wind turbine based on sea test. Ocean Eng 2019;189:106308. 